\title{
A Wireless and Digital Electrode Bus Topology for Biopotential Measurement
}

\author{
Mark Nolan \\ Technological University Dublin, mark.nolan@tudublin.ie \\ Edward Burke \\ Technological University Dublin \\ Eugene Coyle \\ Technological University Dublin
}

Follow this and additional works at: https://arrow.tudublin.ie/teapotcon

Part of the Biomedical Commons, Biomedical Devices and Instrumentation Commons, Digital Communications and Networking Commons, and the Electrical and Electronics Commons

\section{Recommended Citation}

Nolan, P., Burke, E., \& Coule, E. (2012). A Wireless and Digital Electrode Bus Topology for Biopotential Measurement. Irish Signals and Systems Conference (ISSC 2012), NUI Maynooth, Ireland. doi:10.1049/ ic. 2012.0214

This Conference Paper is brought to you for free and open access by the tPOT: People Oriented Technology at ARROW@TU Dublin. It has been accepted for inclusion in Conference Papers by an authorized administrator of ARROW@TU Dublin. For more information, please contact arrow.admin@tudublin.ie, aisling.coyne@tudublin.ie,gerard.connolly@tudublin.ie.

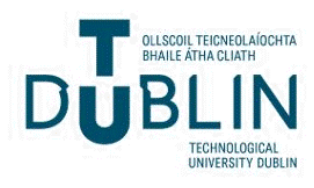




\title{
A Wireless and Digital Electrode Bus Topology for Biopotential Measurement
}

\author{
Mark Nolan", Edward Burke*, Eugene Coyle ${ }^{* *}$ \\ *School of Electrical Engineering Systems \\ **Visiting Fulbright Scholar \\ Dublin Institute of Technology, Dublin 8 \\ Global Policy Research Institute \\ email: mark.nolan@dit.ie \\ Purdue University, Indiana \\ ted.burke@dit.ie \\ email: coylee@purdue.edu
}

\begin{abstract}
The conventional biopotential measurement configuration utilises long lead wires which connect measuring electrodes to signal conditioning circuitry. The majority of bioelectric signals that are measured from the human body have a tiny signal amplitude $(5 \mu \mathrm{V}-5 \mathrm{mV}$ range) and thus any interference that is induced on the lead wires can have a detrimental effect on the original signal.

In this paper, we present an alternative configuration, in which digitisation occurs on the electrode, potentially providing enhanced signal measurement as well as significant benefits in terms of the simplification of the physical interconnections between electrodes. Multiple electrodes are combined to form a digital electrode bus. This proposed topology represents the next stage in the evolution of bioelectric measurement as, due to the decrease in cost and size of integrated circuits, more of the bioinstrumentation circuitry is shifted away from a base measurement station and into the electrode itself.

The prototype wireless device outlined in this paper consists of a grid of electrode nodes. By adding a circuit weighing less than $0.45 \mathrm{~g}$ and with a diameter of $1.5 \mathrm{~cm}$ to standard button clip electrodes, each node contains a high resolution analog-to-digital converter, and a microcontroller. With this design, the lead length has been reduced to a copper PCB track with dimensions of less than $3.5 \times 0.3 \mathrm{~mm}$. All of the nodes are connected onto a shared $\mathrm{I}^{2} \mathrm{C}$ bus on which a master node synchronises slave electrode sampling and coordinates inter-device communication. The system is currently capable of a $215 \mathrm{~Hz}$ sampling rate (for one node at 16-bit resolution) with a limit of up to 79 nodes connected. The system has been demonstrated to work on the electrooculogram and the electrocardiogram and the results for these have been outlined.
\end{abstract}

Keywords - Bioinstrumentation, biomedical electrodes, biomedical electronics, biopotential measurement, wireless body sensor networks.

\section{INTRODUCTION}

In conventional biopotential recording, two or more electrodes are placed on the body and a timevarying electrical potential is recorded. A unipolar lead configuration records the electrical potential at a single point via one signal electrode (relative to a reference potential). A bipolar lead configuration records the electrical potential difference between two points via two signal electrodes. In each case, the signal electrodes are connected to high impedance amplifier inputs, while an additional electrode provides a low-impedance path between the amplifier and human subject. Bipolar leads are usually preferred since interference appearing at both signal electrodes can be eliminated using an instrumentation amplifier with high CMRR.
A drawback of both recording configurations is that lead wires must connect each electrode to the instrumentation equipment. The majority of biopotentials have a tiny signal amplitude $(5 \mu \mathrm{V}-5 \mathrm{mV}$ range) [1]. Consequently, any external electromagnetic interference that is induced on the lead wires can have a corrupting effect on the original measured biopotential signal [2]. High performance modern electrode systems, such as used in the BioSemi Active II [3], go some way towards addressing this problem by incorporating an active element, i.e. operational amplifier, into the electrode. These active electrodes increase the power of the analog biopotential signal relative to any superimposed background noise (i.e. increasing the signal-to-noise ratio). 
A topological evolution, as outlined in this paper, is to eliminate the need for analog-voltage carrying lead wires by digitising biopotentials practically at the point of measurement in an electrode. Several electrodes can then be connected together, via a shared digital bus, to form a body sensor network with numerous advantages over conventional biopotential recording methods. An obvious example is that, due to their nature, conventional bioinstrumentation systems are inherently obtrusive. The system described in this paper reduces the overall lead wiring significantly thus facilitating inconspicuous biopotential recording. The proposed topology represents an evolutionary step in bioelectric signal measurement. Due to the decrease in cost, size and power requirements of integrated circuits, a growing trend is underway whereby the bioinstrumentation circuitry is being shifted away from a base measurement system and into the electrode itself.

Jivet et al. have demonstrated the use of onelectrode integrated circuitry for electrical impedance tomography applications [4, 5]. Their device incorporated on-electrode voltage measuring, constant current generating circuitry and used an $\mathrm{I}^{2} \mathrm{C}$ digital bus and the communication backbone between electrodes. Schnitz designed another example for use in electroencephalogram (EEG) measurement [6]. Their device utilised an SPI digital bus with bus speeds that would allow for a large number of electrodes to be used, as is required with EEG. The examples outlined above form part of a wider body of research in the area of body area networks (BAN) with applications including health care monitoring and human-machine interfaces. The IEEE standard for BANs has been underdevelopment since 2007 [7].

\section{MATERIALS AND METHODS}

\section{a) Signal Conditioning Circuitry}

Fig. 1 illustrates how the system is used to measure the electrocardiogram (ECG) and the electrooculogram (EOG). As shown, all electrode nodes are connected via a common 4-wire bus made of flexible, light-weight and durable wiring. The nodes themselves are built into standard snap-on clips that suit the commonly available button type disposable electrodes.

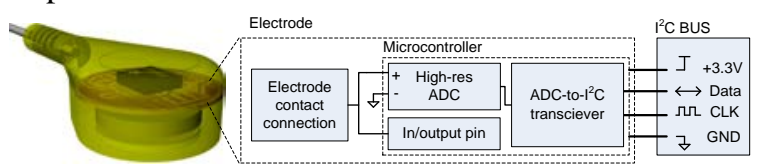

Figure 1: Diagrams illustrating a single digital electrode button clip and a block diagram of the internal configuration.

The positive pin of the differential input to the analog-to-digital converter (ADC) is connected onto the metal of the electrode clip via a short lead wire (3.5x0.3mm). The negative input to the ADC is connected to the circuit ground. As all of the digital electrodes share a floating ground, all measurements are referred to this reference voltage.

The internal electrode configuration in Fig. 1 also shows a general purpose input/output pin (GPIO) tied directly to the electrode. When a biopotential measurement at the electrode is underway, this pin is set as an input (with high input impedance state). Under control of the devices firmware, the pin can be set to be an output and driven low; this has the effect of setting the potential of the electrode in question to be the same as the floating ground. The location of the electrical reference point on the body is therefore digitally selectable.

As stated by Webster [8], "the goal of a biopotential recording system is the faithful reproduction of the signals that are generated in nerve and muscle". With this in mind, careful consideration was given to the electrical properties of the proposed topology. Figure 2 illustrates the equivalent circuit for a three electrode ECG recording.

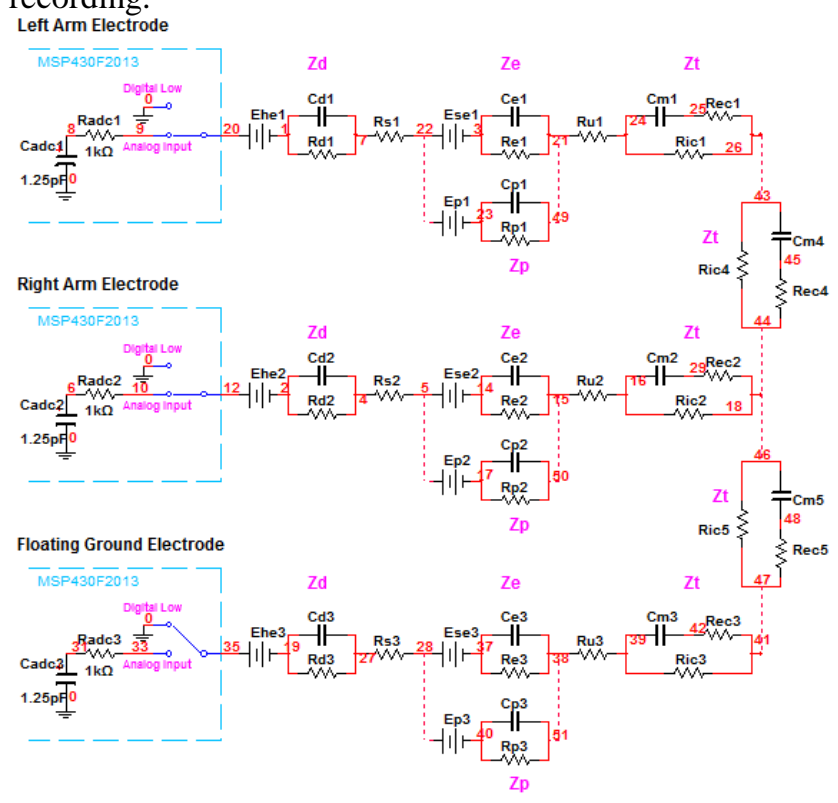

Figure 2: Equivalent circuit model for the instrumentation circuitry, electrode-skin junction and internal body tissue in the current topology. Based on models by [9-11].

This model covers the equivalent impedances for the electrode $Z_{d}$, gel $R_{s}$, skin (epidermis, $Z_{e}$ and sweat glands/ducts, $Z_{p}$ ) and dermis and subcutaneous layers $\mathrm{R}_{\mathrm{u}}$. The DC offsets, $\mathrm{E}_{\mathrm{he}}$ and $\mathrm{E}_{\mathrm{se}}$, are caused by the half cell potentials of the lead-electrode and the electrolyte-skin interface respectively. $Z_{t}$ represents a model for the equivalent circuit for the tissue within a body where the membrane capacitance $\mathrm{C}_{\mathrm{m}}$, intracellular resistance $R_{i c}$ and the extracellular resistance $R_{\mathrm{ec}}$ are shown. The values for the internal input impedance to the sigma-delta $(\Sigma \Delta)$ ADC are given by the manufacturer's specifications. This model attempts to cover the main components in the 
system, however, additional factors such as nuisances with relation to different tissue types or electrode printed circuit board dynamics are not represented.

\section{b) Transmission Bus}

Several desirable features have been incorporated into the design of the digital electrode bus. The predominant feature was the minimisation of the number of wires shared between all electrodes. Another attribute was that the data bus would be required to have a sufficient bandwidth so as to be capable of fast data transmission rate to allow for an adequate sampling rate from each sensor. For both of these reasons, the $\mathrm{I}^{2} \mathrm{C}$ data bus topology was chosen as the basis for the device described in this paper.

Each electrode node is connected on a fourwire bus which comprises of a shared clock, data and power supply connections (positive and ground). The $\mathrm{I}^{2} \mathrm{C}$ data bus is set to run at a $400 \mathrm{kHz}$ clock speed although, depending on the length of the bus wires, the actual clock frequency can vary. One microcontroller, a Texas Instruments (TI) MSP430F2132, was set as a master and it coordinated communication between it and multiple slave TI MSP430F2013 microcontrollers. As the current system uses 7-bit addressing, a theoretical maximum of 112 nodes $(128-16$ reserved addresses) could be connected on the bus. In practice, however, $\mathrm{I}^{2} \mathrm{C}$ specifications limit the bus capacitance to $400 \mathrm{pF}$ [12] and by taking into account the bus capacitance of master $(\approx 5 \mathrm{pF})$, the slaves $(\approx 5 \mathrm{pF}$ each) and the physical transmission medium, this number is reduced to a maximum of 79 nodes.

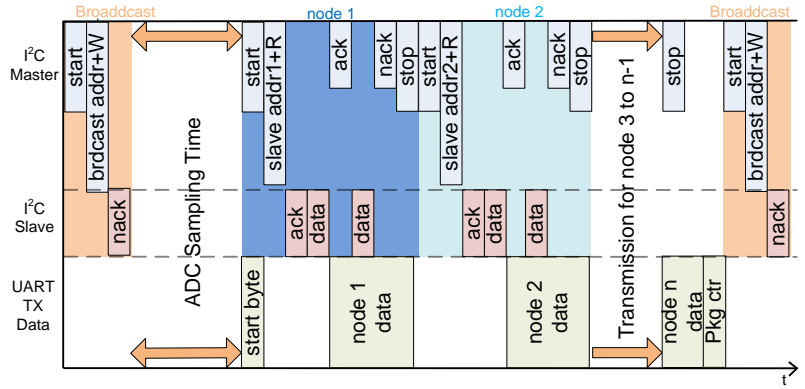

Figure 3: Sequence diagram of data transmission for a multiple node system whereby the $\mathrm{n}^{\text {th }}$ node is set as a ground reference. (Note: time axis is not to scale)

At the start of each transmission, the master sends out a broadcast address. This signal is used to synchronise the slaves so that all recording nodes sample their ADCs simultaneously. Upon reception of this signal by each of the slaves, all activity on the bus goes silent for a set duration of time. This delay is required by the $\Sigma \Delta \mathrm{ADCs}$; it allows the voltage under measurement to settle before a sample is recorded. The master then polls each of the nodes individually for their respective data. As the master receives the data samples, it simultaneously transfers this data to an internal universal asynchronous

receiver/transmitter (UART) which is connected to a serial-to-Bluetooth converter. Utilising a Bluetooth enabled computer, the digital electrode bus appears as a virtual serial port from which the stream of data is read. A virtual instrument was created in LabVIEW to parse, store and graphically display the data stream.

\section{c) Sampling Rate}

The maximum sampling frequency that the digital electrode bus can facilitate is limited by a number of factors of which the principle factors are listed below in order of impact.
1. ADC sampling time
4. UART baud rate
2. Number of nodes
3. $\mathrm{I}^{2} \mathrm{C}$ clock speed
5. Internal $\Sigma \Delta \mathrm{ADC}$ gain setting

The predominant limiting factor on the sampling rate of the current digital electrode grid is governed by a hardware property of the $\Sigma \Delta$ ADC. To make use of the full range of the 16-bit resolution a long sampling time is required $(\approx 4.1 \mathrm{~ms})$.

Due to the polling nature of the $\mathrm{I}^{2} \mathrm{C}$ data bus, each sensor takes it in turn to transmit its data back to the master. Therefore, the greater the number of nodes, the larger the overall data packet length and the longer the transmission time per packet. The hardware specific limited speeds of $400 \mathrm{kHz}$ for $\mathrm{I}^{2} \mathrm{C}$ and 460800 bps for the UART link follow next. The value for the $\mathrm{I}^{2} \mathrm{C}$ pull-up resistors was carefully chosen at $1 \mathrm{k} \Omega$ with the aim of complementing the value of the bus capacitance and its possible variation between different recording topologies.

The resistor-capacitor (RC) network on the input to the ADC as illustrated in Figure 2, also puts a limit on the sampling frequency. The values of the $\mathrm{RC}$ components are given by TI and are dependent on the internal gain setting of the $\Sigma \Delta \mathrm{ADC}$.

\section{d) Experimental Setup}

To gauge the performance of the system a 3 electrode ECG and a 5 electrode EOG were recorded on a healthy male subject. Although, in this case, the electrode bus is measuring two particular biosignals, the system can be easily adapted to measure a range of bioelectrical phenomena.

(a)
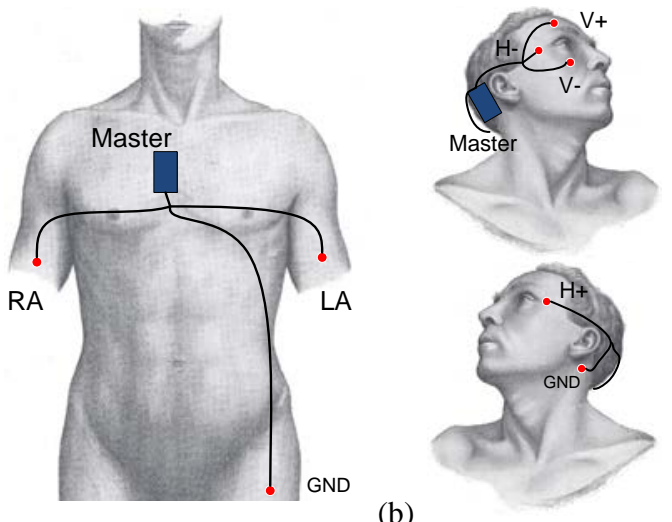

(b)

(a) 3 : Electrode placement for (a) 3 electe ECG and (b) 5 electrode EOG. Images modified from [13] 
Figure 4 illustrates the electrode placement for each of the experimental setups. Each of the electrode nodes where attached to the skin using standard disposable snap-on silver/silver chloride electrodes. During measurement, the Bluetooth enabled master device was placed in a neck lanyard for ECG and an over-the-ear bracket for EOG.

\section{RESULTS \\ a) $E O G$}

The digital electrodes were placed on unprepared skin in the configuration shown in Figure 4 (b). The subject was asked to move their eyes backwards and forth to the extreme position on the horizontal and vertical planes. Equations (1) and (2) were solved to find the voltage difference between each pair of electrodes. Figure 5 shows the resulting waveforms from three full vertical movements followed by three full horizontal movements.

$$
\text { Horizontal Potential }=H^{+}-H^{-}
$$

Vertical Potential $=V^{+}-V^{-}$

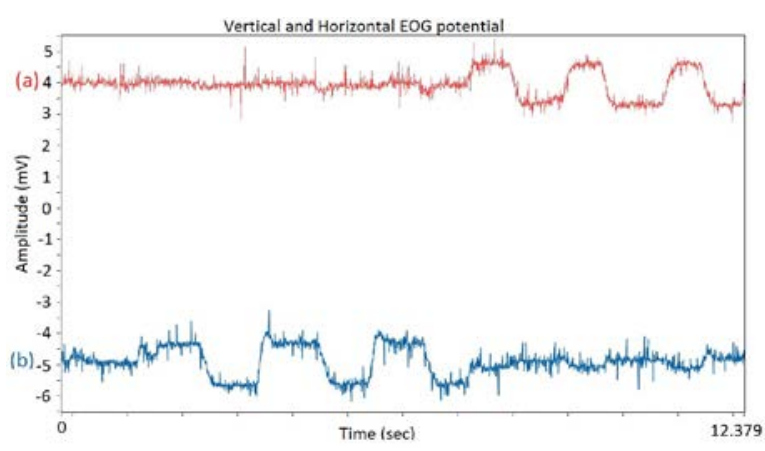

Figure 5: A 12.379 sec window of unfiltered EOG activity.

(a) Horizontal potential. (b) Vertical potential.

\section{b) ECG}

As with the EOG experiment, the digital electrodes were placed on unprepared skin in the configuration shown in Figure 4 (a). The data was processed in LabVIEW and Figure 6 shows the lead I voltage potential given by the following equation.

$$
\text { Lead } I=L A-R A
$$

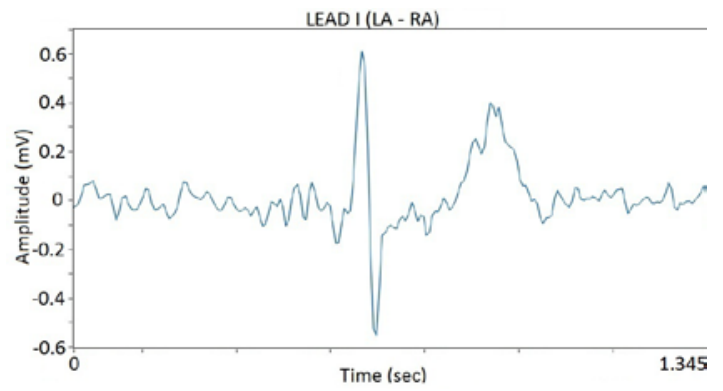

Figure 6: A 1.345 sec window of lead I ECG activity showing the PQRST cardiac cycle. Gain is set at 32.

\section{DISCUSSION}

A novel body area network was designed with several features that advance the state of the art in biopotential measurement. A key benefit of the system is in its interoperability. It has a standard hardware and software architecture, as facilitated by the $\mathrm{I}^{2} \mathrm{C}$ communication standard, which allows for a multitude of possible sensors to be added to a single recording data bus. Recent revisions of the $\mathrm{I}^{2} \mathrm{C}$ protocol list advances which could be incorporated into the system such as 16-bit addressing and a nine fold increase in the data throughput to $3.4 \mathrm{Mbits} / \mathrm{s}$. The described topology could also facilitate possible use in a large cross-sectional area biosignal sensing system by using a 'blanket' grid of electrodes with a digitally selectable reference point.

Further investigation is required to facilitate a full comparison between the novel device described and established techniques. Issues such as ADC matching and susceptibility to external electrical noise may be of concern and should form part of this investigation. It is envisaged that each electrode will be rigorously characterised so that their individual electrical characteristics can be ascertained and compensated for.

\section{REFERENCES}

[1] W. H. Olson, "Basic Concepts of Medical Instrumentation," in Medical Instrumentation: Application and Design, J. G. Webster, Ed., 4th ed New York: John Wiley \& Sons, 2009, pp. 1-44.

[2] J. C. Huhta and J. G. Webster, "60-Hz Interference in Electrocardiography," Biomedical Engineering, IEEE Transactions on, vol. BME-20, pp. 91-101, 1973.

[3] Biosemi. (2002, 14th April 2012). Available: http://www.biosemi.com/products.htm

[4] I. Jivet, "Architecture of an on Electrode Integrated Electronics with an All Digital Interface for Electrical Impedance Tomography," in International Conference on Advancements of Medicine and Health Care through Technology, 2009, pp. 205-208.

[5] I. Jivet and B. Dragoi, "On-electrode autonomous current generator for multi-frequency EIT," Physiological Measurement, vol. 29, p. S193, 2008.

[6] B. A. Schnitz, J. A. Stewart, R. V. Allen, and K. C. Fadem, "Improving Signal Quality and Test Reliability in EEG Measurements Using Integrated High-Density Surface-Mount Electronics," in Medical Electronics Symposium Proceedings, 2004.

[7] Institute of Electrical and Electronics Engineers. IEEE 802.15 WPAN. Available: http://www.ieee802.org/15/pub/TG6.html

[8] J. G. Webster, "Reducing Motion Artifacts and Interference in Biopotential Recording " IEEE Transactions on Biomedical Engineering, vol. BME31, pp. 823-826, Dec. 1984.

[9] Texas Instruments. (2004). MSP430x2xx Family User's Guide (Rev. $\quad I \quad$ ed.). Available: http://www.ti.com/product/msp430f2013

[10] M. R. Neuman, "Biopotential Electrodes," in Medical Instrumentation: Application and Design, J. G. Webster, Ed., 4th ed New York: John Wiley \& Sons, 2009, pp. 189-240.

[11] K. S. Cole and R. H. Cole, "Dispersion and Absorption in Dielectrics I. Alternating Current Characteristics," Journal of Chemical Physics, vol. 9, p. 11, 1941.

[12] NXP Semiconductors. (2012, 13th February 2012). I2C-bus specification and user manual. Available: http://www.nxp.com/documents/user manual/UM1020 4.pdf

[13] H. Gray, Anatomy of the Human Body, 20th Edition ed.: Philadelphia: Lea \& Febiger, 1918. 